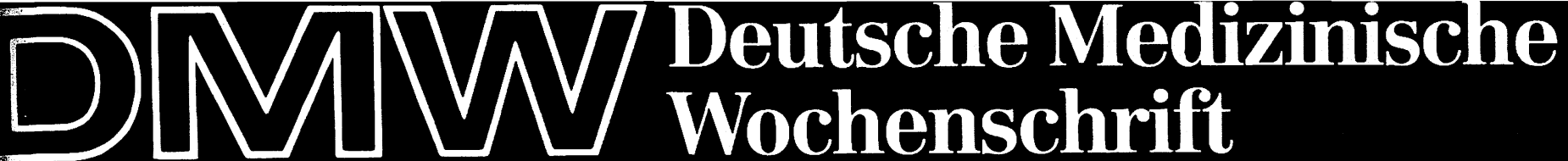

P. C. Scriba, München

W. Siegenthaler, Zürich

A. Sturm, Herne/Bochum
F. Kümmerle, Mainz

R. Augustin, Stuttgart

W. Kuhn, Stuttgart

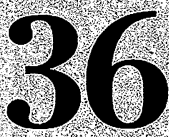

115. Jahrgang

7. Sept 1990

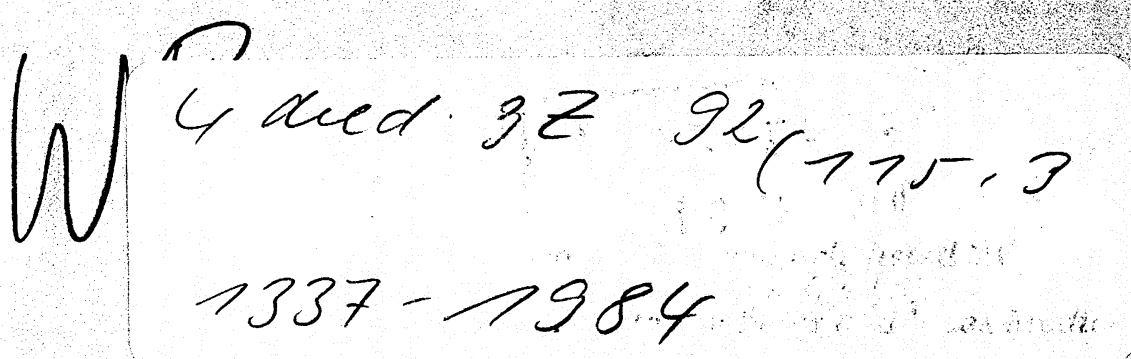

Mediquiz 1337

Originalien

Endosonographische Diagnostik bei

Pankreastumoren 1339

\section{urze Originalien \& Fallberichte}

Zervikale Myelopathie bei Mucopolysaccharidose

Typ II 1348

Disseminierte Histoplasmose als Erstmanifestation einer HIV-Infektion 1353

\section{Ktuelle Diagnostik \& Therapie}

Kernspintomographie bei Verletzungen des Kniegelenks 1358

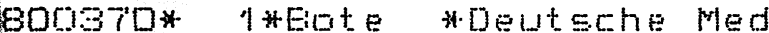

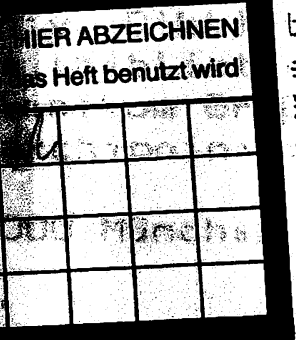

thet: pinchen

It: Medizin

inadern

$\therefore=15$

$7 \mathrm{O}$

\section{Übersichten}

Bildgebende Diagnostik bei Erkrankungen der

Bauchspeicheldrüse 1361

\section{Arztrecht in der Praxis}

Behandlung durch einen selbständig handelnden Assistenzarzt (Anfängeroperation) 1368

\section{Fragen aus der Praxis}

Nachsorge bei operierter Jodmangelstruma

\section{Leser-Zuschriften}

Das mitochondriale Genom und seine

Deletionen 1373

\section{Kleine Mitteilungen}

Diphosphonat-Therapie der postmenopausalen Osteoporose 1374

Riesenzellarteriitis 1375

Vegetarische Kost 1375

Jeder Zwölfte ist schwerbehindert 1375

Hochschulnachrichten 1376 


\title{
Erfolgreiche medikamentöse Therapie einer Aspergillen-Endokarditis
}

\author{
J. R. Bogner, S. Lüftl, M. Middeke und F. Spengel \\ Medizinische Poliklinik (Vorstand: Prof. Dr. N. Zöllner) der Universität München
}

\begin{abstract}
Bei einem 74jährigen Patienten, der über 3 Monate $7 \mathrm{~kg}$ an Gewicht abgenommen sowie an Fieber bis $38^{\circ} \mathrm{C}$ und Anämie (Hämoglobin $9,4 \mathrm{~g} / \mathrm{dl}$ ) gelitten hatte, kam es zu Schmerzen und Blaufärbung der Finger II-V der rechten Hand. Die Echokardiographie ergab Vegetationen auf der Aortenklappe. In einer Blutkultur wuchs Aspergillus fumigatus. Daraufhin wurde die Diagnose einer Aspergillen-Endokarditis gestellt. Prädisponierende Faktoren für diese Erkrankung wurden nicht gefunden. Aufgrund des Alters und der Begleiterkrankungen des Patienten (arterielle Verschlußkrankheit, Zustand nach Hinterwandinfarkt) wurde von einem Herzklappenersatz abgesehen und eine Therapie mit Amphotericin B intravenös (in steigender Dosierung bis $50 \mathrm{mg} / \mathrm{d}$ ) und $1,5 \mathrm{~g} / \mathrm{d}$ Flucytosin oral eingeleitet. Im Laufe der fünfwöchigen Therapie mit insgesamt $1,1 \mathrm{~g}$ Amphotericin B und 41,5 g Flucytosin gingen die Beschwerden und die Klappen-Vegetationen zurück, und der Patient ist seit nunmehr 9 Monaten rezidivfrei.

\section{Successful drug treatment of aspergillus endocarditis}

A 74-year-old man who had a weight loss of $7 \mathrm{~kg}$ in three months, with fever up to $38^{\circ} \mathrm{C}$ and anaemia $(\mathrm{Hb} 9.4 \mathrm{~g} / \mathrm{dl})$ began to have pain and blue discoloration of fingers II-V of the right hand. Echocardiography demonstrated vegetation on the aortic valve cusps and blood culture grew Aspergillus fumigatus, indicating aspergillus endocarditis. There were no predisposing factors. Valve replacement was contraindicated because of the age of the patient, the presence of peripheral arterial disease, and previous myocardial infarction. Treatment was started with amphotericin B i.v. (dosage increasing to $50 \mathrm{mg}$ daily) and $1.5 \mathrm{~g}$ daily of flucytosine by mouth, to a total of $1.1 \mathrm{~g}$ amphotericin $\mathrm{B}$ and $41.5 \mathrm{~g}$ flucytosine in five weeks. During this time there was a gradual decrease in symptoms and the valve vegetations. Nine months later there has been no recurrence.
\end{abstract}

Die durch Aspergillen verursachte Endokarditis wurde zuerst von Zimmerman (24) im Jahre 1950 beschrieben. Sie macht weniger als 0,5\% aller Endokarditiden aus (15); die Inzidenz hat jedoch - wie auch die Häufigkeit anderer Aspergillosen $(4,10)$ - in den letzten Jahren zugenommen $(2$, $8,14,23)$. Fast immer sind prädisponierende Faktoren vorhanden, wie vorausgegangene offene Operationen am Herzen, eine das Immunsystem schwächende Grundkrankheit, vorausgehende Steroid-, Zytostatika- oder Antibiotikatherapie sowie intravenöse Drogenabhängigkeit. Unbehandelt führt die Erkrankung stets zum Tode, aber auch mit Therapie ist die Prognose meist infaust $(2,8,14,23)$.

Dtsch. med. Wschr. 115 (1990), 1833-1837

(c) Georg Thieme Verlag Stuttgart $\cdot$ New York
Im folgenden berichten wir über einen Patienten mit Aspergillen-Endokarditis ohne erkennbare prädisponierende Faktoren, bei dem eine alleinige medikamentöse Behandlung erfolgreich war.

\section{Kasuistik}

Anamnese. Der 74jährige Patient mit koronarer Herzkrankheit, Hinterwandinfarkt vor 10 Jahren, Claudicatio intermittens mit Schmerzen im linken Unterschenkel nach einer Gehstrecke von $100 \mathrm{~m}$ und rezidivierenden Keratitiden hatte in den letzten 3 Monaten etwa $7 \mathrm{~kg}$ an Gewicht abgenommen sowie an Inappetenz, Abgeschlagenheit und Fieber um $38^{\circ} \mathrm{C}$ gelitten. 5 Tage nach der stationären Aufnahme in einem auswärtigen Krankenhaus traten Schmerzen und eine Blauverfärbung der Finger II-V der rechten Hand auf. Daraufhin wurde über 24 Stunden über einen A.-brachialis-Katheter eine Lysetherapie mit 20000 I.E./h Urokinase durchgeführt. Nach weiteren 3 Tagen kam es zusätzlich zu Schmerzen und Blauverfärbung der linken Zeigefingerkuppe. Daraufhin wurde der Patient in unsere Klinik verlegt. 
Körperliche Untersuchung. Der Patient befand sich in reduziertem Allgemeinzustand (Gewicht $63 \mathrm{~kg}$, Größe $167 \mathrm{~cm})$. Der Puls war regelmäßig mit 100 Schlägen pro Minute, der Blutdruck betrug 160/100 mm Hg. Der Patient wies einen Faßthorax auf mit hypersonorem Klopfschall beiderseits sowie Knistern und feinblasigen Rasselgeräuschen rechts basal. Das Herz war auskultatorisch unauffällig. Die Bauchdecken waren weich, der Leberdurchmesser betrug $10 \mathrm{~cm}$ in der Medioklavikularlinie, und die Untersuchung von Milz und Nierenlagern ergab keinen pathologischen Befund. Die A. dorsalis pedis links und beide Arteriae tibiales posteriores waren nicht tastbar. Der Triceps-surae-Reflex war an beiden Beinen nicht auslösbar; ansonsten war die neurologische Untersuchung unauffällig. Die Untersuchung der Lymphknoten ergab keinen pathologischen Befund. An den Fingern II-V rechts fiel eine blau-livide Verfärbung auf. Diese Bereiche waren kalt und sehr druckschmerzhaft. Am Rand des Nagelfalzes des rechten Zeigefingers war ein stecknadelkopfgroßer blauer Punkt sichtbar.

Klinisch-chemische Befunde. Die Blutsenkung war mit $60 / 82 \mathrm{~mm}$ stark beschleunigt. Das kleine Blutbild ergab eine mikrozytäre Anämie mit einem Hämoglobingehalt von $9,4 \mathrm{~g} / \mathrm{dl}$ und eine Leukozytose von $14900 / \mu \mathrm{l}$. Im Differentialblutbild wurden $1 \%$ Stabkernige, $75 \%$ Segmentkernige, $16 \%$ Lymphozyten, $5 \%$ Monozyten, 1\% Myelozyten und 2\% Metamyelozyten nachgewiesen. In der Serumelektrophorese fiel eine Erhöhung der $\alpha_{1}$ - und $\alpha_{2}$-Globuline (7,4 bzw. 16,4\%) auf. Die Serum-Konzentrationen betrugen für Kreatinin $1,2 \mathrm{mg} / \mathrm{dl}$, für Harnstoff- $\mathrm{N} 41 \mathrm{mg} / \mathrm{dl}$, für Eisen $49 \mu \mathrm{g} / \mathrm{dl}$ und für Ferritin $1034 \mu \mathrm{g} / \mathrm{l}$. Alle weiteren klinisch-chemischen Befunde lagen im jeweiligen Referenzbereich.

Der klinische Verlauf und die bis zu diesem Zeitpunkt vorhandenen Befunde ergaben somit ein Krankheitsbild mit rezidivierenden arteriellen Embolien und erheblichen Entzündungszeichen. Differentialdiagnostisch kam daher am ehesten eine entzündliche Veränderung des proximalen Aortenabschnitts oder eine Endokarditis in Frage.

Apparative Verfahren. Die daraufhin durchgeführte Echokardiographie zeigte Vegetationen auf der Aortenklappe (Abbildung 1) ohne Hinweis auf eine Klappeninsuffizienz. Die Größenverhältnisse der Herzkammern waren normal. Im Hinterwandbereich waren Bewegungsstörungen zu erkennen

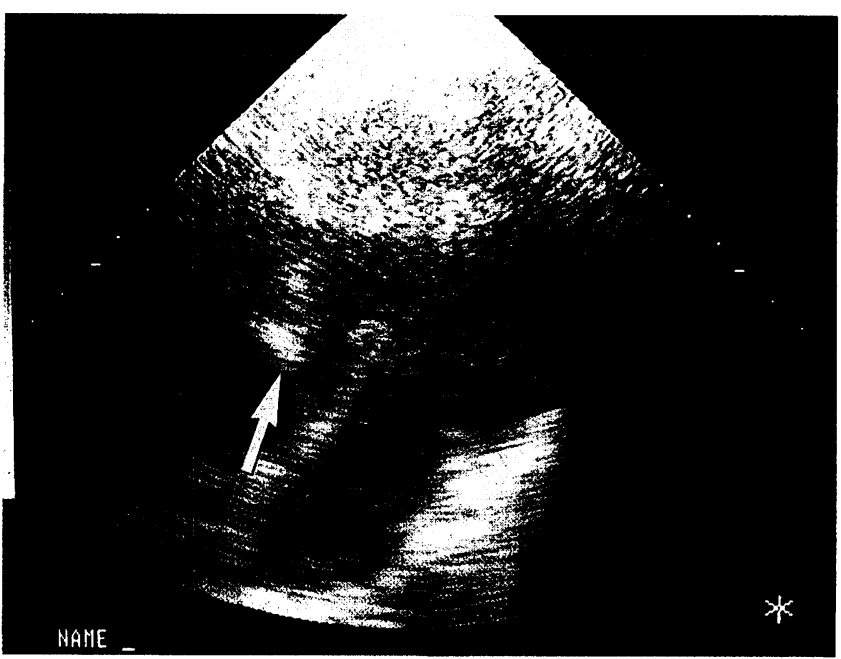

Abb. 1 Echokardiographischer Befund: Vegetation auf der Aortenklappe (Pfeil).
Wegen der blau-lividen Verfärbung der Finger der rechten Hand wurde eine Brachialis-Angiographie in DSA-Technik vorgenommen. Es fanden sich Kaliberunregelmäßigkeiten der Arteriae radialis und ulnaris sowie ein embolischer Verschluß der A. radialis in Höhe des Daumensattelgelenks rechts. Der Arcus palmaris profundus war nur inkomplett, die Gefäße im Bereich des zweiten und dritten Fingers waren gar nicht darstellbar.

Im Röntgenbild des Thorax zeigte sich ein flächiges Infiltrat rechts basal. Das Elektrokardiogramm war, von Hinweisen auf den alten Hinterwandinfarkt abgesehen, unauffällig, und die Oberbauchsonographie ergab keinen pathologischen Befund.

Therapie und Verlauf. Wegen des Verdachts auf eine arterielle Embolie erhielt der Patient zunächst 1100 I.E./h Heparin intravenös. Um eine möglichst optimale rheologische Therapie der verbleibenden Endstrombahn der rechten Hand zu gewährleisten, wurden zusätzlich für 7 Tage täglich $60 \mu \mathrm{g}$ Alprostadil in $500 \mathrm{ml}$ physiologischer Kochsalzlösung über 4 Stunden infundiert. Da sich in der Echokardiographie Vegetationen auf der Aortenklappe darstellten, wurde der Patient unter der Verdachtsdiagnose einer Endokarditis bei zunächst negativen Blutkulturen ungezielt mit Penicillin (dreimal täglich 10 Mega I.E. intravenös) und Tobramycin (dreimal täglich $40 \mathrm{mg}$ intravenös) behandelt. Dieses Vorgehen zeigte keine Wirkung. 4 Tage nach Therapiebeginn erlitt der Patient, wohl aufgrund einer Embolie einen apoplektischen Insult mit Eintrübung, motorischer Aphasie und Hemiballismus rechts. In der kranialen Computertomographie ergab sich kein Anhalt auf eine frische intrazerebrale Blutung oder ein Infarktareal.

Zwischenzeitlich konnte aus einer Blutkultur Aspergillus fumigatus isoliert werden. Die daraufhin eingeleitete antimykotische Behandlung bestand aus einer Kombination von Amphotericin B und Flucytosin. Nach einer Testdosis von $0,1 \mathrm{mg} / \mathrm{kg}$ Körpergewicht wurden in steigender Dosierung bis $50 \mathrm{mg} / \mathrm{d}$ (entsprechend $0,8 \mathrm{mg} / \mathrm{kg}$ ) Amphotericin B lichtgeschützt infundiert sowie $1,5 \mathrm{~g} / \mathrm{d}$ Flucytosin oral gegeben. Wegen des schlechten Allgemeinzustandes und des hohen Alters des Patienten mußte von einem operativen Herzklappenersatz abgesehen werden. Unter der antimykotischen Therapie nahmen die Herzklappenauflagerungen ab. Die Echokardiographie zeigte 4 Tage nach Beginn der antimykotischen Behandlung keine zusammenhängenden Vegetatio-

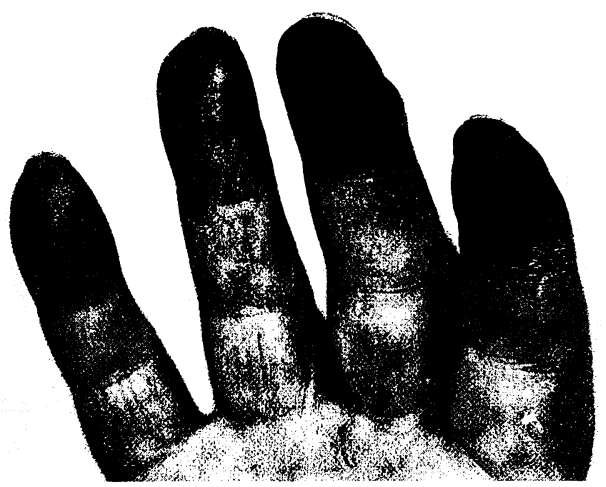

Abb. 2 Überwiegend trockene Gangrän des zweiten und dritten Fingers der rechten Hand. 
nen mehr, sondern ließ mehrere getrennte Echomuster erkennen. Die neurologische Symptomatik bildete sich binnen einer Woche zurück. Die Endglieder des rechten Zeige- und Mittelfingers demarkierten sich zunehmend, und es entstand eine trockene Gangrän (Abbildung 2). Die einen Monat nach der stationären Aufnahme erforderliche Fingerteilamputation führte zu einem funktionell guten Ergebnis (Abbildung 3). Aspergillen ließen sich im Amputat nicht nachweisen. Die antimykotische Behandlung wurde über 5 Wochen bis zu einer Amphotericin-B- und Flucytosin-Gesamtdosis von $1,1 \mathrm{bzw}$. $41,5 \mathrm{~g}$ fortgeführt, und die Heparinisierung wurde während des gesamten Krankheitsverlaufs beibehalten. Dabei ergab sich kein Hinweis auf weitere Embolien. Bis auf eine reversible Erhöhung der Nierenretentionswerte (Serum-Kreatininkonzentration $2,3 \mathrm{mg} / \mathrm{dl}$ ) und eine substitutionsbedürftige Hypokaliämie wurde die antimykotische Therapie komplikationslos vertragen.

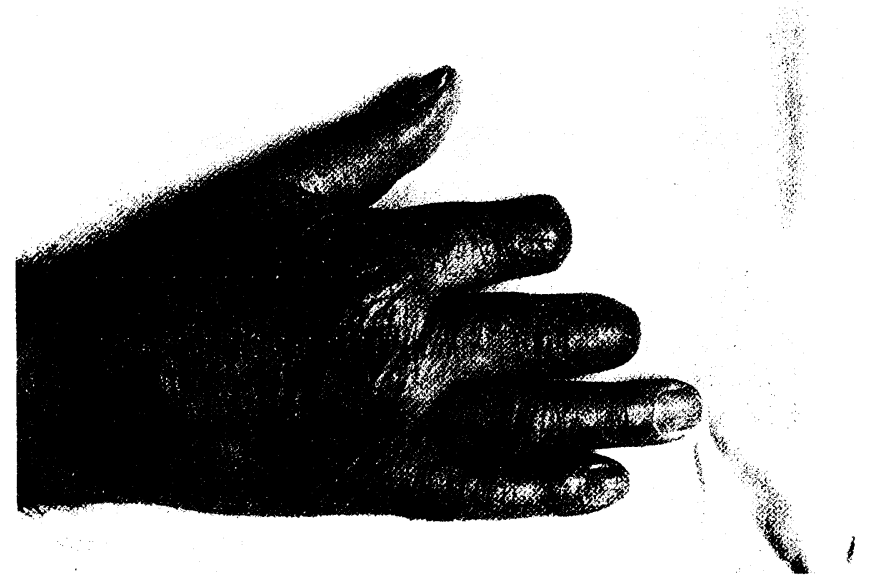

Abb. 3 Ergebnis nach Fingerteilamputation des zweiten und dritten Fingers der rechten Hand.

Zur Klärung einer der Aspergillen-Endokarditits zugrundeliegenden Erkrankung wurde der Patient gründlich untersucht. Es zeigte sich eine Störung der zellulären Immunität mit kutaner Anergie im Hauttest. Bei einer Lymphopenie bis minimal 3\% ergab die Analyse der Lymphozyten-Subpopulationen eine normale Verteilung von Helfer- und Suppressorzellen. Die Untersuchung auf Antikörper gegen das Human-Immunodeficiency-Virus war negativ. Für EpsteinBarr-Virus ergab sich ein Durchseuchungstiter. Sämtliche immunserologischen Untersuchungen erbrachten einen negativen Befund.

Eine umfangreiche Tumorsuche, einschließlich Koloskopie und Knochenmarksbiopsie, blieb ohne Hinweis auf ein Malignom. Die Ursache des Lungeninfiltrats konnte zwar nicht endgültig geklärt werden, computertomographisch ergab sich jedoch kein Anhalt für einen pulmonalen oder mediastinalen Tumor.

7 Wochen nach der stationären Aufnahme konnte der Patient beschwerdefrei entlassen werden. Im weiteren Verlauf ergab sich bei engmaschigen ambulanten Kontrollen kein Hinweis auf ein Rezidiv der Erkrankung oder erneute Embolien. Der Patient war 9 Monate nach der Entlassung in gutem Allgemeinzustand. Er hatte $8 \mathrm{~kg}$ an Gewicht zugenommen und fühlte sich gesund. Echokardiographisch ließen sich keine Herzklappenvegetationen mehr darstellen, und das Lungeninfiltrat war vollständig resorbiert.

\section{Diskussion}

Der Krankheitsverlauf bei unserem Patienten ist in dreierlei Hinsicht ungewöhnlich. Die Aspergillen-Endokarditis trat ohne erkennbare prädisponierende Faktoren auf, der Pilz wurde ante mortem aus einer Blutkultur isoliert, und die Erkrankung konnte durch alleinige antimykotische Therapie klinisch geheilt werden.

$\mathrm{Zu}$ den prädisponierenden Faktoren der Aspergillen-Endokarditis zählen in der Reihenfolge ihrer Häufigkeit: Operationen am offenen Herzen, länger andauernde Steroid- oder Antibiotikabehandlung, hämatologische Malignome, Zytostatikabehandlung und seltenere Ursachen wie intravenöse Drogenabhängigkeit, Herzkatheteruntersuchungen und Organtransplantationen (23). In einzelnen Kasuistiken wurden Aspergillen-Endokarditiden auch nach Herzschrittmacher-Implantation beschrieben $(1,9)$. Nur in 12-15\% aller Fälle tritt die Erkrankung, wie bei unserem Patienten, ohne erkennbare prädisponierende Faktoren auf $(8,23)$.

Ein direkter Erregernachweis gelingt bei Aspergillen-Endokarditiden ante mortem nur in weniger als $25 \%$ aller Fälle. Wegweisend ist zumeist die histologische und kulturelle Aufarbeitung von Embolektomie-Material oder entfernten Herzklappen. Die Häufigkeitsangabe für Aspergillen-Wachstum in Blutkulturen schwankt zwischen 0 (23) und $8 \%$ (8). Die wenigen positiven Blutkulturen benötigten zudem eine lange Bebrütungszeit - zwischen 14 und 25 Tagen - bis zur Diagnose $(8,15)$. Bei unserem Patienten war die Blutkultur schon nach 6 Tagen positiv.

Große Bedeutung bei der Diagnose einer Endokarditis hat in den letzten beiden Jahrzehnten die Echokardiographie erlangt. Die typischen Herzklappenauflagerungen können zumeist gut erkannt werden. Auch bei unserem Patienten ließen sich deutliche Vegetationen auf der Aortenklappe darstellen. Trotzdem darf ein negativer echokardiographischer Befund nicht überbewertet werden. Es gibt mehrere Berichte $(7,12,21)$ über falsch-negative Ergebnisse bei subvalvulärer Pilzendokarditis. Serologische Untersuchungen auf Aspergillen-Antikörper $(3,13)$ konnten sich bislang nicht durchsetzen.

Die Prognose der Aspergillen-Endokarditis ist sehr schlecht. Die hohe Sterblichkeit ist zum Teil auch durch die Grundkrankheit und die Toxizität der Antimykotika bedingt (15). Nur in Einzelfällen wurde über eine erfolgreiche Therapie berichtet $(21,22)$. In zwei Übersichtsartikeln liegt die Heilungsrate zwischen $5 \%$ (8) und $10 \%$ (23). Berichtet wird dabei jeweils nur über ein chirurgisches Vorgehen mit Herzklappenersatz oder eine kombinierte chirurgisch-medikamentöse Behandlung. 
Über den therapeutischen Erfolg einer ausschließlich medikamentösen Behandlung ist unseres Wissens bislang nicht berichtet worden.

Unser Patient wies fast alle Hauptsymptome einer Aspergillen-Endokarditis auf: Fieber, Anämie, Embolien in die beiden am häufigsten betroffenen Regionen - Zentralnervensystem und Extremitäten - sowie Gewichtsverlust $(8,23)$. An wesentlichen Befunden fehlte lediglich ein Herzgeräusch; dieses tritt aber bei $40-50 \%$ aller Erkrankten nicht auf $(8,23)$. Das Lungeninfiltrat läßt sich gut mit der Diagnose vereinbaren: In einer Übersicht (23) wurden bei $33 \%$ der Patienten fokale Infiltrate beschrieben. Der echokardiographische Befund stützt die Diagnose einer Endokarditis, ohne freilich Auskunft über den Erreger geben zu können. Das Aspergillenwachstum konnte nur in einer von fünf entnommenen Blutkulturen nachgewiesen werden. Ein Artefakt ist jedoch unwahrscheinlich, da eine akzidentelle Verunreinigung mit Aspergillen sehr ungewöhnlich ist und sich auch kein Hinweis auf eine Kontamination im Laboratorium ergab. Für die Richtigkeit der Diagnose sprechen schließlich der Rückgang der Aortenklappenvegetationen und die prompte Besserung der neurologischen Symptomatik unter antimykotischer Therapie.

Unklar bleibt die Genese der rezidivierenden Keratitiden in der Vorgeschichte. Ein Erregernachweis war damals nicht geführt worden. Mykotische Hornhautentzündungen sind in unseren Breiten jedenfalls sehr selten; als Erreger sind dabei aber Aspergillen am häufigsten $(5,20)$.

Zur Beurteilung des therapeutischen Erfolges gibt es in der Literatur keine allgemein anerkannten Richtlinien. Unser Patient konnte beschwerdefrei entlassen werden. Er hatte kein Fieber mehr, die Anämie war zurückgegangen, und es traten keine weiteren Embolien auf. Bei der Echokardiographie konnten 9 Monate nach der Entlassung aus der stationären Behandlung keine Vegetationen der Aortenklappe mehr erkannt werden, und das Lungeninfiltrat war vollständig resorbiert worden. In den 40 Wochen seit Absetzen der antimykotischen Therapie hat sich kein Hinweis auf ein Rezidiv ergeben.

Nachdem bei unserem Patienten keine Infektionsmöglichkeit durch invasive Verfahren, wie offene Herzoperation, Herzkatheterismus oder Schrittmacherimplantation, bestanden hatte, stellte sich die Frage nach dem Infektionsweg. In mehreren Arbeiten wurde auf die fast ubiquitäre Verbreitung von Aspergillenkonidien hingewiesen $(6,16,19,23)$. Vor allem im häuslichen Wohnbereich wird die Raumluft häufig durch Konidienreservoirs in Topfpflanzenerde kontaminiert. Weitere häufige Streuherde sind Nüsse, Kleintiere, Tierfutter, Luftbefeuchter und Pilzwachstum bei bautechnischen Mängeln, zum Beispiel feuchten Räumen (19). Auch unser
Patient hatte in seinem Wohnzimmer mehrere Topfpflanzen, so daß eine aerogene Inokulation mit nachfolgender hämatogener Streuung als der wahrscheinlichste Infektionsweg anzusehen ist (11). Eine weitgehende Expositionsprophylaxe im häuslichen Bereich läßt sich kaum durchsetzen und erscheint auch nicht sinnvoll, da Pilzkonidien für Gesunde apathogen sind. Im Krankenhaus jedoch müssen immungeschwächte Patienten vor Pilzinfektionen geschützt werden $(10,17)$. Als wirkungsvoll und wenig aufwendig erweisen sich bereits so einfache Maßnahmen wie der Ersatz von Topfpflanzen durch gut gepflegte Hydrokulturen (18).

\section{Literatur}

1 Acquati, F., F. Semeraro, E. Respighi, R. Gallotti, S. Repetto, G. Binaghi: Aspergillus flavus-infection of a pacemaker wire. Continuing evidence for active management of infected pacemarkers. G. ital. Cardiol. 17 (1987), 467.

2 Atkinson, J. B., D. H. Connor, M. Robinowitz, H. A. McAllister, R. Virmani: Cardiac fungal infections. Review of autopsy findings in 60 patients. Hum. Path. 15 (1984), 935.

3 Bennett, J. E.: Rapid diagnosis of candidiasis and aspergillosis. Rev. infect. Dis. 9 (1987), 398.

4 Boeckh, M., G. Höffken, H. Lode: Spektrum pulmonaler Manifestationen von Aspergillosen. Dtsch. med. Wschr. 114 (1989), 1706.

5 Böke, W.: Entzündungen der Hornhaut. In François, J., F. Hollwich (Hrsg.): Augenheilkunde in Klinik und Praxis, Bd. II (Thieme: Stuttgart 1981), 1.31.

6 Corrigan, C., S. M. Horner: Aspergillus endocarditis in association with a false aortic aneurysm. Clin. Cardiol. 11 (1988), 430.

7 McFadden, P. M., L. Gonzalez-Lavin, J. S. Remingston: Limited reliability of the "negative« two-dimensional echocardiogram in the evaluation of infectious vegetative endocarditis. Diagnostic and surgical implications. J. cardiovasc. Surg. 26 (1985), 59.

8 Kammer, R. B., J. P. Utz: Aspergillus species endocarditis. The new face of a not so rare disease. Amer. J. Med. 56 (1974), 506.

9 Kramer, L., R. R. Rojas-Corona, D. Sheff, E. S. Eisenberg: Disseminated aspergillosis and pacemaker endocarditis. Pacemaker 8 (1985), 225.

10 Lie, T., S. M. Höfer, C. Höhnke, L. Krizek, E. Kühnen, A. Iwatnscheff, O. Köster, A. Overlack, J. Vogel, K. Rommelsheim: Aspergillose nach Lebertransplantation als Hospitalismusinfektion. Dtsch. med. Wschr. 112 (1987), 297.

11 Nishiura, T., Y. Miyazaki, K. Oritani, N. Tominaga, Y. Tomiyama, S. Katagiri. Y. Kanayama, T. Yonezawa, S. Tarui, T. Yamada, M. Sakurai, H. Kume, M. Okudaira: Aspergillus vegetative endocarditis complicated with schizocytic hemolytic anemia in a patient with acute lymphocytic leukemia. Acta haemat. (Basel) 76 (1986), 60.

12 Peterson, S. P., N. Schiller, R. B. Stricker: Failure of twodimensional echocardiography to detect aspergillus endocarditis. Chest 85 (1984), 291.

13 DeRepentigny, L., M. Boushira, L. Ste-Marie, G. Bosisio: Detection of galactomannan antigenemia by enzyme immunoassay in experimental invasive aspergillosis. J. clin. Microbiol. 25 (1987), 863.

14 Rubinstein, E., E. R. Noriega, M. S. Simberkoff, R. Holzman, J. J. Rahal: Fungal endocarditis. Analysis of 24 cases and review of the literature. Medicine (Baltimore) 54 (1975), 331.

15 Schmidt, D., F. Nager: Endokarditis mit ungewöhnlichen Erregern. Schweiz. med. Wschr. 117 (1987), 2097.

16 Staib, F.: Raumluftuntersuchung auf Aspergillus-Arten in der Wohnung eines chronisch Lungenkranken. Bundesgesundheitsblatt 21 (1978), 471.

17 Staib, F.: Zur Bekämpfung von Aspergillus-fumigatus-Infektionen während des Klinikaufenthalts. Öff. Gesundh.-Wes. 41 (1979), 777. 
18 Staib, F., C. Rajendran: Untersuchungen von HydrokulturZimmerpflanzen auf menschenpathogene Aspergillus-Arten. Hyg. u. Med. 5 (1980), 575.

19 Staib, F.: Mykosen durch Pilzsporen in der Raumluft. Zbl. Bakt., 1. Abt. Orig. B 176 (1982), 142.

20 Sundaram, B. M., S. Bandrinath, S. Subramanian: Studies on mycotic keratitis. Mycoses 32 (1989), 568.

21 Swennson, E. E., V. L. Willman, G. J. Peterson: Acute aortic occlusion from aspergillosis in a healthy patient with survival. J. vasc. Surg. 4 (1986), 187.

22 Wagner, D. K., P. H. Werner, L. I. Bonchek, T. Shimshak, M. W. Rytel: Successful treatment of post-mitral valve annuloplasty aspergillus flavus endocarditis. Amer. J. Med. 79 (1985), 777.

23 Woods, G. L., R. P. Wood, B. W. Shaw: Aspergillus endocarditis in patients without prior cardiovascular surgery. Report of a case in a liver transplant recipient and review. Rev. infect. Dis. 11 (1989), 263.

24 Zimmerman, L. E.: Candida and aspergillus endocarditis. With comments on the role of antibiotics in dissemination of fungus disease. Arch. Path. 50 (1950), 591.
Dr. J. R. Bogner, S. Lüftl, Privatdozent Dr. M. Middeke, Prof. Dr. F. Spengel

Medizinische Poliklinik der Universität

Pettenkoferstr. 8a

W-8000 München 2 